\title{
LUT
}

Lappeenranta

University of Technology

\section{Numerical study on the effect of cross-section orientation on fluid flow and heat transfer in a periodic serpentine triangular microchannel}

Filimonov Roman, Sorvari Joonas

This is an Final draft version of an article
published by Elsevier
in Applied Thermal Engineering

DOI: $\quad$ 10.1016/j.applthermaleng.2017.07.027

Copyright of the original publication: (c) Elsevier 2017

Please cite the publication as follows:

Filimonov, R., Sorvari, J. (2017). Numerical study on the effect of cross-section orientation on fluid flow and heat transfer in a periodic serpentine triangular microchannel. Applied Thermal Engineering, Vol 125, Oct 2017. p. 366-376. 10.1016/j.applthermaleng.2017.07.027

This is a parallel published version of an original article. This version can differ from the original published article. 


\title{
Numerical study on the effect of cross-section orientation on fluid flow and heat transfer in a periodic serpentine triangular microchannel
}

\author{
Roman Filimonov*, Joonas Sorvari \\ Lappeenranta University of Technology, School of Engineering Science, P.O. Box 20, \\ FIN-53851 Lappeenranta, Finland
}

\begin{abstract}
The influence of cross-section orientation on fluid flow and heat transfer in a periodic serpentine equilateral triangular microchannel was investigated by means of computational fluid dynamics (CFD) modeling. Serpentine channels with cross-sections of upward- and left-pointing equilateral triangles were considered. Serpentine channels with twisted sections of different degrees of twisting were also considered and evaluated. The simulations were performed for fullydeveloped periodic flow with constant properties under constant wall heat flux (H2) thermal boundary conditions at Reynolds numbers of 50, 100 and 150 (corresponding to Dean numbers of 38, 76 and 114). The results showed that the heat transfer performance of the triangular channel is sensitive to the orientation of its cross-section. At all the Reynolds numbers studied, the channel with left-pointing triangular cross-section provided a higher mean heat transfer enhancement than the channel with upward-pointing cross-section, whereas the pressure drop remained the same. It was also found that by employing twisted shapes, it is possible to rotate the cross-section into a more favorable orientation for local heat transfer and thus improve the overall thermal performance further. With a moderate pressure drop increase, mean heat transfer enhancements for the channels with twisted sections were found to be greater than those
\end{abstract}

\footnotetext{
${ }^{*}$ Corresponding author. Tel.: +358 414968098.

Email address: roman.filimonov@lut.fi (Roman Filimonov)
}

Postprint submitted to Applied Thermal Engineering 
for non-twisted configurations over almost the whole range of Reynolds numbers considered. However, the mean heat transfer enhancement did not rise with increasing degree of twisting, although the pressure drop increased with the degree of twisting.

Keywords: Heat transfer enhancement, Serpentine microchannel, Twisted channel, Triangular cross-section, Pressure drop, CFD.

\section{Introduction}

Heat transfer enhancement in microchannels has received increasing attention due to the rapid development of microscale heat transfer devices for various applications such as electronics cooling, microreactors and transport of living

5 cells [1]. The flow regime in microstructures is usually laminar, and heat transfer, compared to turbulent conditions, is thus significantly reduced. To improve heat transfer in microchannels, various enhancement techniques have been developed. They can be classified into active and passive depending on the operation principle. Active techniques use external sources such as vibration or electrohydrodynamic, to enhance heat transfer, while passive techniques typically rely on channel geometry or fluid additives [2]. However, active techniques, which can provide considerable enhancement of heat transfer, need additional power supply and control units, and hence might require complex fabrication and assembly [3].

Compared to active methods, passive techniques, which also can substantially augment heat transfer performance, have been studied more widely due to the advantages of ease of fabrication, higher reliability and relatively low cost. Heat transfer enhancement with passive techniques usually can be achieved by using different channel shapes, structural materials and working fluids [4]. Ghani

20 et al. [5] provided a comprehensive review on the effects of microchannel design on the heat transfer performance. The authors considered various passive enhancement techniques based on modified channel geometry. Some of the channel modifications discussed in their work are curved channels, channels with ribs, 
grooves or combination of them, and offset strip fins. Although the heat transfer enhancement by such modifications can be significant, it is accompanied by an increase in pressure drop as compared to straight channel. It is reported that curved channels have been shown to be one of the most effective configurations in improving heat transfer performance. Using serpentine configurations, a considerable heat transfer augmentation with moderate pressure drop can be reached.

30 When a fluid flows through a curved channel, secondary vortices are formed around the bends disrupting the thermal boundary layer and thus improving the heat transfer performance. Ghani an co-authors particularly emphasized that a combination between different heat transfer enhancement techniques can have more advantages than a single technique alone.

$35 \quad$ Fluid flow and heat transfer in serpentine microchannels have been studied extensively. Geyer et al. [6] and Karale et al. [7] investigated the effect of various geometric parameters and Reynolds numbers on the heat transfer performance of curved channels with semi-circular and rectangular cross-sections respectively. In their works, the channel shapes varied from a regular serpentine with smooth bends to a zig-zag pathway with sharp corners. Sui et al. [8] and Mohammed et al. [9] analyzed numerically wavy microchannels with rectangular cross-section. The influence of geometric parameters on the heat transfer efficiency at various Reynolds numbers was also considered in their studies. Rosaguti et al. [10], Geyer et al. [11] and Gupta et al. [12] performed simulations of fluid flow 45 and heat transfer in microchannels with, correspondingly, circular, square and triangular cross-sections following the same serpentine path. Silva et al. [13] studied the thermal performance of curved channels with elliptic cross-section. All the aforementioned studies report substantial heat transfer enhancement in the curved channels relative to straight channels with identical cross-sections.

Twisted channels is another geometric configuration that has been used to improve heat transfer performance by means of producing secondary flows. Tan et al. [14] and Bhadouriya et al. [15] carried out experimental and numerical studies of fluid flow and heat transfer in twisted centimeter-scale ducts with, respectively, oval and square cross-sections. Yang et al. [16] conducted 
experimental investigation on flow and heat transfer characteristics of twisted centimeter-scale elliptical channels. The impact of geometric dimensions and Reynolds number was analyzed in these works. Fluid flow and heat transfer in twisted milli-scale channels with different cross-sections including circular, semi-circular, elliptic, square, rectangular and triangular were studied via numerical simulations by Khoshvaght-Aliabadi and Arani-Lahtari [17, 18]. They particularly examined the effect of various working fluids. The results of these studies indicate that twisted channels can also provide significantly higher heat transfer efficiency than straight smooth channels.

Although both serpentine and twisted types of channels have demonstrated improved heat transfer capabilities compared to straight channels, their thermal efficiency has been shown to be strongly dependent on the geometric parameters and Reynolds number $[17,19]$. Therefore, great attention has been paid to study of the influence of geometric characteristics such as channel pathway $[6,7]$, crosssection shape $[12,17]$ and twist pitch length $[14,16]$ over various Reynolds number ranges.

However, practically no emphasis has been given to the effect of cross-section orientation on local heat transfer performance in serpentine channels. A change of the orientation of some cross-sections such as triangular cross-sections, for example, can cause variations of flow distribution around the bends. Hence,

75 the local heat transfer coefficient can change as well. In this work, serpentine microchannels with equilateral triangular cross-section are considered. Fluid flow and heat transfer characteristics for different cross-section orientations are numerically investigated over a range of Reynolds numbers. The concept of utilizing twisted shapes in serpentine microchannels as a mean of further increasing of the heat transfer performance is proposed.

\section{Numerical analysis}

The microchannels under consideration have a periodic geometry comprising repeating serpentine units. A schematic diagram of the axial path of the unit 
is presented in Figure 1. The serpentine path is defined as follows: $S / H=1$,

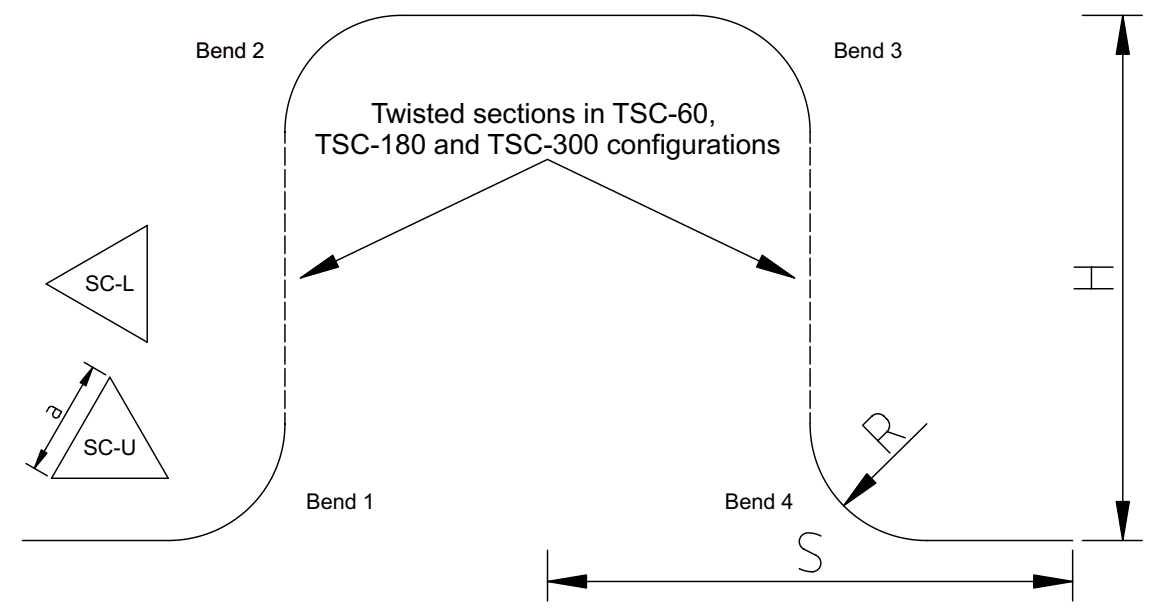

Figure 1: Axial path of the serpentine unit.

${ }_{85} S / a=4.5$ and $R / a=1$, where $S$ is the unit half length, $H$ is the unit height, $a$ is the cross-section side length, and $R$ is the radius of curvature of the bends. The given serpentine path is similar to that studied in [12]. Different channel configurations were considered, depending on the cross-section orientation. SC-U and SC-L types are serpentine channels with cross-sections of upward90 and left-pointing equilateral triangles. TSC configuration corresponds to the SC-U type with uniformly twisted vertical channel sections indicated in Figure 1. The twisting changes the cross-section orientation from upward-pointing to downward-pointing and back by rotating the cross-section around the axial direction. The center of the rotation of the cross-section coincides with the circumcenter of the cross-section. Based on the degree of twisting, three configurations are distinguished: TSC-60, TSC-180 and TSC-300 with $60^{\circ}, 180^{\circ}$ and $300^{\circ}$ twists, respectively. All the described configurations are summarized in Table 1. Figure 2 illustrates some of the generated computational domains used for the CFD analysis.

100

The computational domains were meshed with a structured grid in ANSYS ICEM 15.0 by sweeping the cross-sectional grid consisting of mainly quadrilat- 
Table 1: Channel configurations.

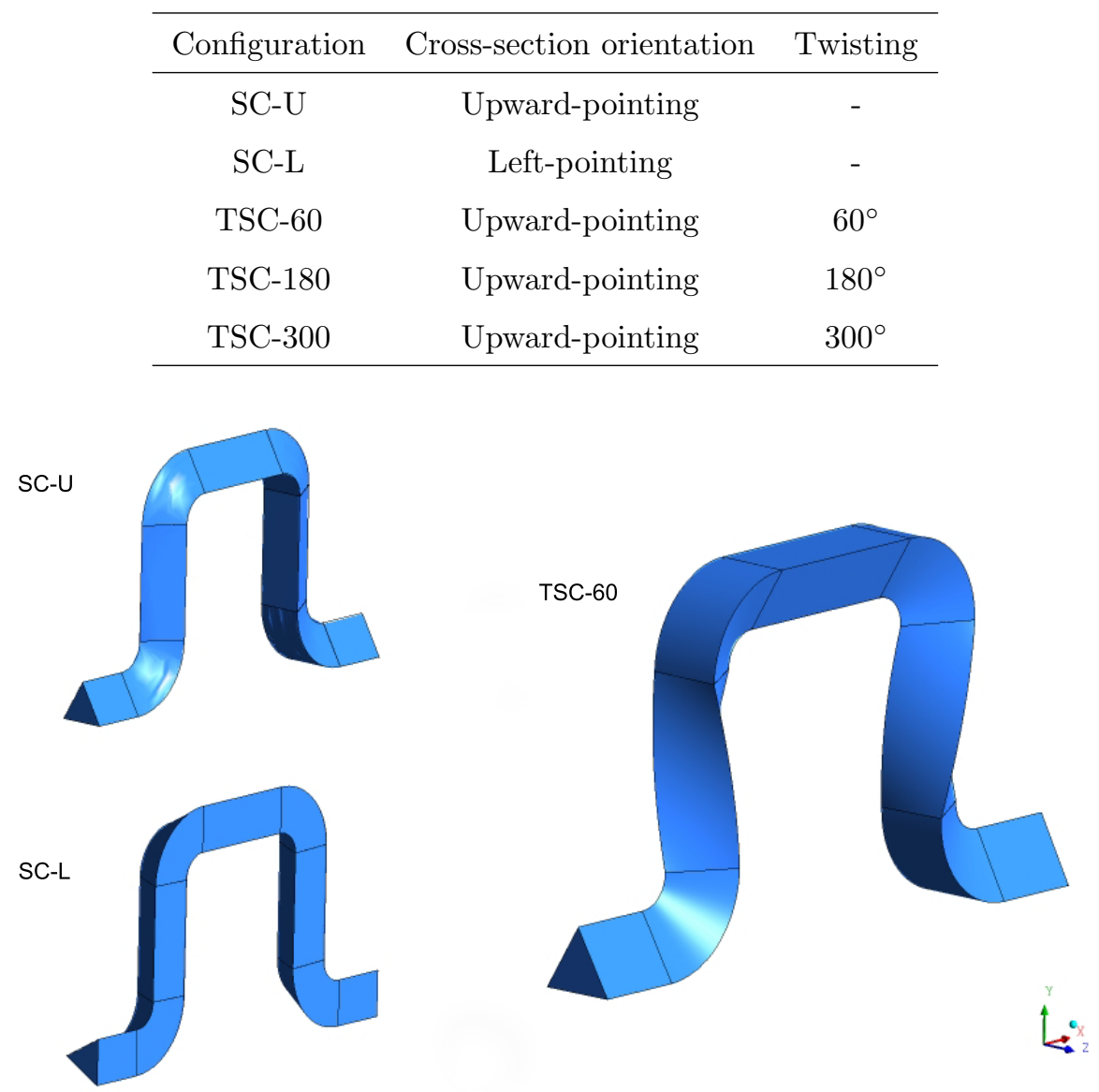

Figure 2: Computational domains used for CFD analysis.

eral elements along the channel path shown in Figure 1. A finer mesh resolution was applied in the near wall region, where the velocity and temperature gradients are largest. The axial grid spacing was uniform, with slightly smaller spacing around the bends and in the twisted sections.

After a sufficiently long distance from the inlet, depending on the geometry and Reynolds number, the velocity distribution and non-dimensional temperature pattern in periodic geometry become constant for each repeating module, and the flow is then said to be hydrodynamically and thermally fully-developed. 
where $N u_{H 2, s e r}, f_{\text {ser }}, N u_{H 2, s t r}$ and $f_{\text {str }}$ are Nusselt numbers and Fanning friction factors in the serpentine and straight channels, respectively. For lamiet al. [20] was therefore used in this study. Due to the periodicity, only a single unit was modeled. The inlet and outlet of the unit were considered as periodic planes.

Fluent 15.0 software was utilized to perform numerical simulations of the periodic flow and heat transfer. The fluid flow, namely water flow, was first calculated by solving three-dimensional steady incompressible mass and momentum conservation equations. A constant mass flow rate corresponding to $R e=50,100$ or 150 was defined at the inlet, and no-slip boundary conditions were assigned to the walls of the channel. The gravitational force was neglected in the simulations. Then, when the velocity field was obtained, the energy equation was solved alone to predict the temperature field. Constant wall heat flux (H2 type [21]) thermal boundary conditions were set at the wall boundaries. The working fluid properties were assumed constant (Prandtl number of 6.13) and temperature-independent. The Semi-Implicit method for Pressure-Linked (SIMPLE) algorithm for pressure-velocity coupling and second-order upwind scheme for the discretization of the convection terms in the solved equations were used in the numerical calculations. More details about the governing equations, numerical methods and techniques applied in this study can be found in [20], the Fluent Theory Guide [22] and Fluent Users Guide [23].

The performance of the serpentine channels was assessed by comparing such characteristics as Nusselt number and friction factor to those for a straight channel with identical cross-section under the same flow and thermal conditions. Then, heat transfer enhancement factor, $e_{N u_{H} 2}$, and pressure drop penalty factor, $e_{f}$, can be expressed as

$$
e_{N u_{H 2}}=\frac{N u_{H 2, s e r}}{N u_{H 2, s t r}}
$$

$$
e_{f}=\frac{f_{\text {ser }}}{f_{\text {str }}}
$$


nar fully-developed flow in a straight channel with equilateral triangular crosssection, $N u_{H 2, s t r}$ and $f_{\text {str }} \cdot R e$ are constant and equal, respectively, to 1.892 and 13.333 [21].

The overall performance of each channel configuration was evaluated using a performance evaluation criterion (PEC) [24]

$$
E=\frac{\bar{e}_{N u_{H 2}}}{e_{f}^{1 / 3}}
$$

The Nusselt number at a given axial position $l$ was evaluated using the peripherally-averaged heat transfer coefficient, which is defined as

$$
h_{l}=\frac{q_{w}}{\bar{T}_{w}-T_{b}}
$$

145

\section{Results and discussion}

Grids of different resolutions were tested to determine the appropriate crosssectional and axial meshes for the calculations. The mesh sensitivity test was performed by modeling periodic flow and heat transfer in a straight channel with equilateral triangular cross-section at $R e=150$. The computed values of 
Table 2: $N u_{H 2}$ and $f \cdot R e$ for different cross-sectional mesh refinements.

\begin{tabular}{cccc}
\hline Mesh configuration & Number of cross-sectional elements & $N u_{H 2}$ & $f \cdot R e$ \\
\hline 1 & 1900 & 1.915 & 13.300 \\
2 & 2500 & 1.910 & 13.306 \\
3 & 3300 & 1.909 & 13.312 \\
\hline
\end{tabular}

$N u_{H 2}$ and $f \cdot R e$ for different cross-sectional mesh sizes are given in Table 2. The computed $N u_{H 2}$ and $f \cdot R e$ on the grid from the mesh configuration 1 varied by $0.26 \%$ and $0.04 \%$, respectively, from those for the mesh configuration 2 . On the grids from the mesh configurations 2 and 3, the corresponding differences were $0.05 \%$ and $0.04 \%$ respectively. The cross-sectional grid from the mesh configuration 2 was thus selected as the optimal for further simulations. The difference between the values for Nusselt number and friction factor obtained with the mesh configuration 2 and the corresponding analytical values given in [21] is less than $1 \%$. The axial path was divided into approximately $160-180$ element groups to ensure mesh independent results, as it was determined in [12]. Figure 3 illustrates the optimal mesh configuration.
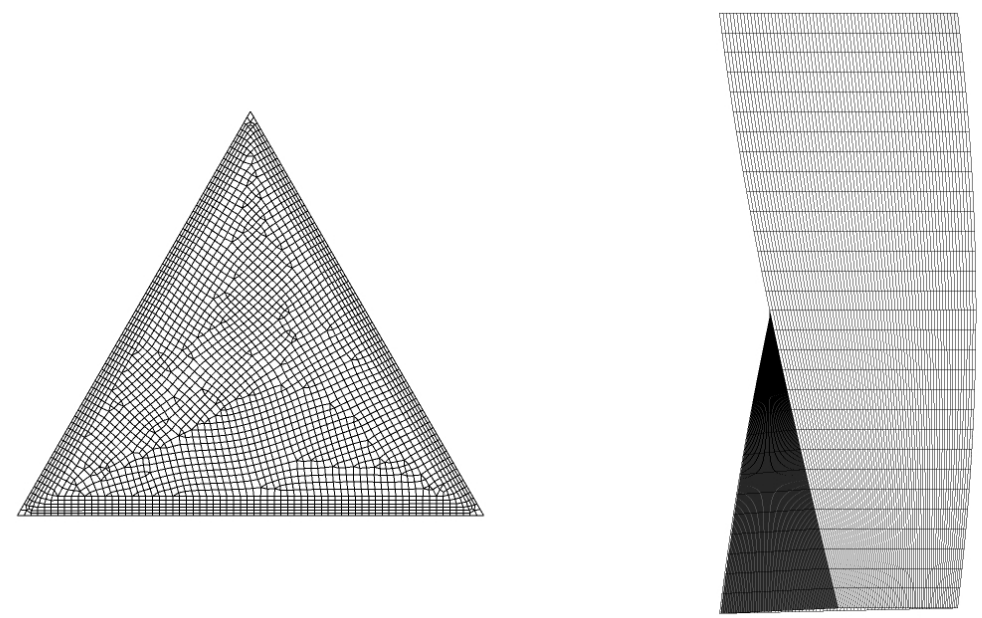

Figure 3: Cross-sectional (left) and axial (right) grids. 
To additionally validate the numerical approach, results on heat transfer enhancement and pressure drop penalty for the SC-U channel were compared with those obtained by Gupta et al. [12]. Reynolds numbers of 25 and 200 were also included to the validation. The values in the present simulations matched the previous numerical data well enough, see Figure 4.

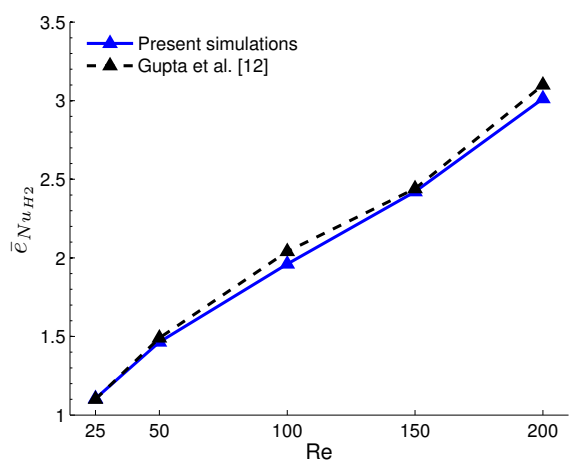

(a)

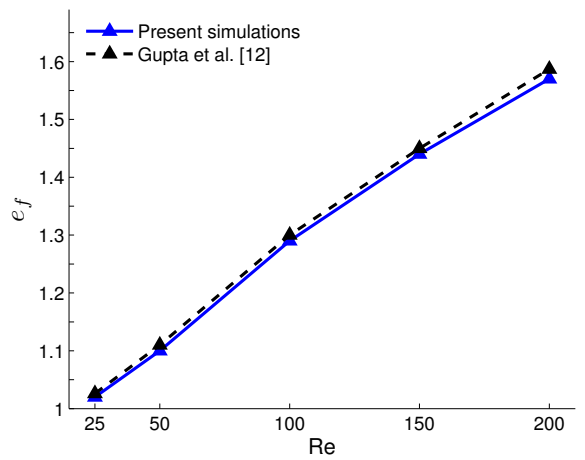

(b)

Figure 4: Comparison of simulated and literature [12] values for mean heat transfer enhancement (a) and pressure drop penalty (b) for channels with equilateral triangular cross-section following the same serpentine path for different Reynolds numbers.

Simulation results obtained for the non-twisted configurations are compared first. Figure 5 shows the variation of heat transfer enhancement across the units for different Reynolds numbers. The heat transfer performance increases with the $R e$ value for both channel types. As can be seen in Figure 5, an increment 


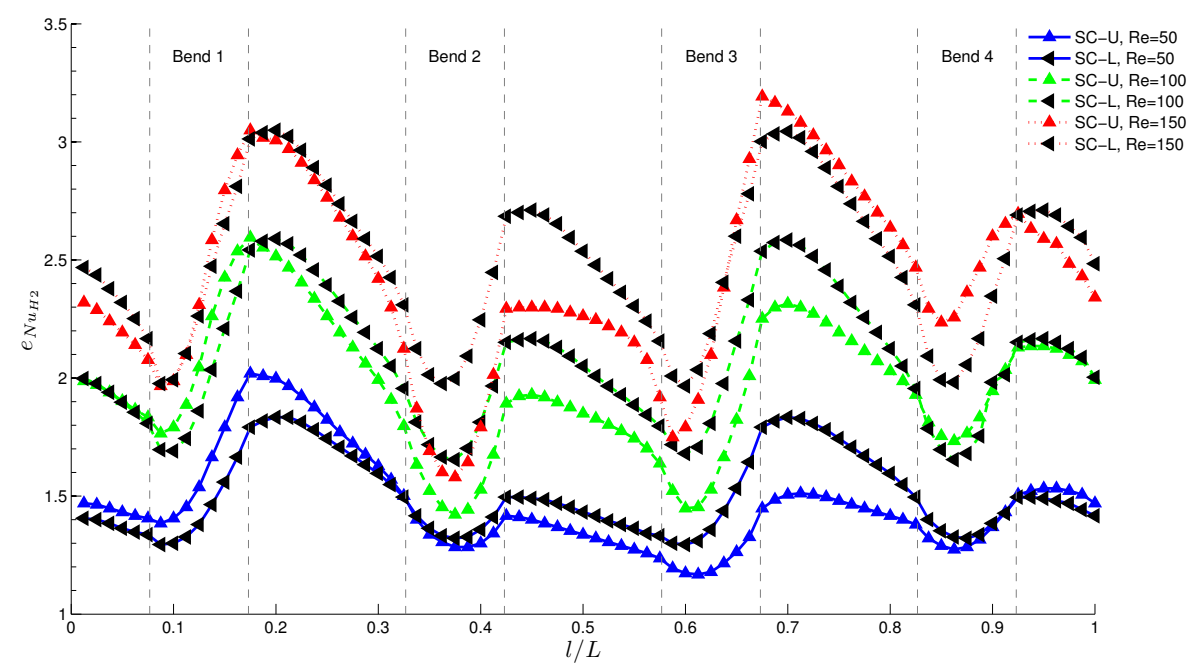

Figure 5: Heat transfer enhancement in the SC-U and SC-L configurations as a function of normalized axial distance at Reynolds numbers of 50,100 and 150. The intervals bounded by a pair of dashed vertical lines correspond to bend sections.

of $e_{N u_{H} 2}$ is observed as the flow passes through each bend, with peak values at the exit of the bends. At higher flow velocities, the intensity of counter-rotating vortices, called Dean vortices [25] and shown in Figure 6, becomes stronger, and hence more cold fluid is transported from the center of the channel to the walls, resulting in improved heat transfer rates. Dean [25] characterized such vortices by a non-dimensional parameter, called Dean number, $R e \sqrt{D_{h} / R}$. For the channel configurations considered in this study, the Dean number is equal to approximately $0.76 \cdot R e$.

The heat transfer enhancement is greater after Bend 1 and Bend 3 than after Bend 2 and Bend 4, as observed in Figure 5. It is caused by a change of the rotation direction of Dean vortices due to the changing direction of rotation of the bends [10]. At $R e=50$ and 100, the heat transfer augmentation after Bends 1 and 3 of the SC-L channel type is approximately at the same level, whereas for the SC-U configuration, the heat transfer rate after Bend 1 is higher than that after Bend 3. In particular, the SC-L geometry shows a noticeably stronger heat transfer augmentation after Bend 3 compared to that in the SC- 

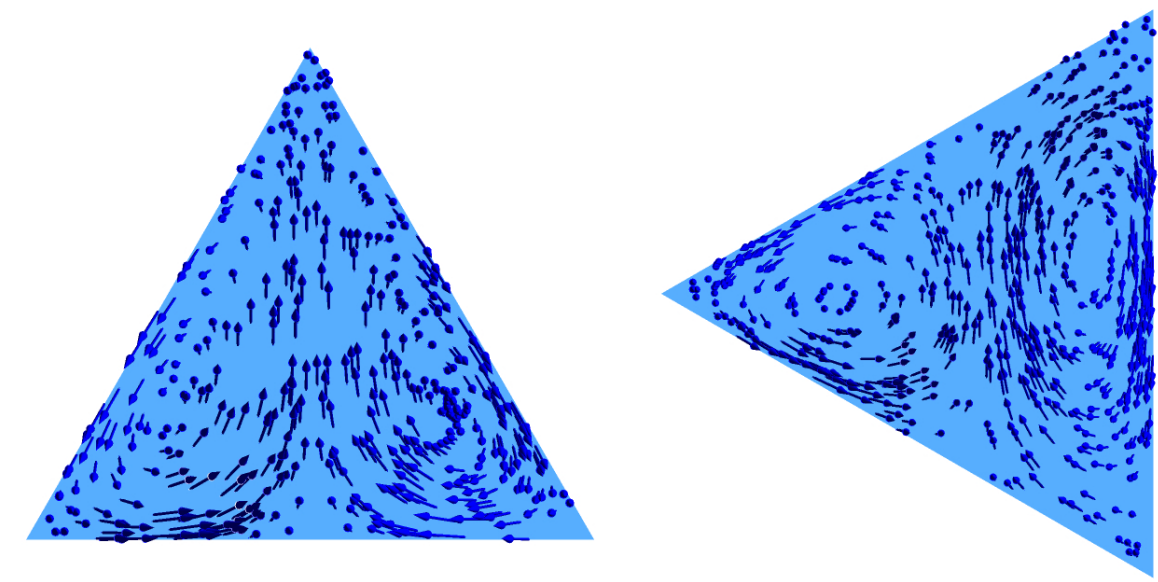

Figure 6: Secondary flows in the form of two counter-rotating vortices, known as Dean vortices, at the exit of the Bend 2 of the SC-U (left) and SC-L (right) channels.

$\mathrm{U}$ case. However, at $R e=150$, heat transfer in Bend 3 of the SC-U channel increases considerably, reaching over three times greater performance at the exit than the corresponding straight channel and exceeding the peak $e_{N u_{H 2}}$ of the SC-L configuration at the same location. The performance in Bend 2 of the SC-L channel is higher at all the Reynolds numbers considered, whereas it is roughly similar in Bend 1 and Bend 4, compared to the SC-U configuration. With the same pressure drop as the SC-U channel, the SC-L configuration demonstrates $2.7 \%, 5.6 \%$ and $2.9 \%$ higher mean heat transfer enhancement at Reynolds numbers of 50, 100 and 150 respectively.

Although the mean heat transfer performance of the SC-L configuration does not increase significantly, local variations across the unit are noticeable. The effect of cross-section orientation is most pronounced for Bends 2 and 3. A centrifugal acceleration induced by the curvature forces the fluid to move toward the outer channel region. Thus, when the flow passes through Bends 2 and 3, the SC-L configuration provides better overall contact between the cold fluid and the channel boundary and the thinner thermal boundary layer, as illustrated in Figure 7, and hence improved heat transfer. Likewise, the heat transfer in 

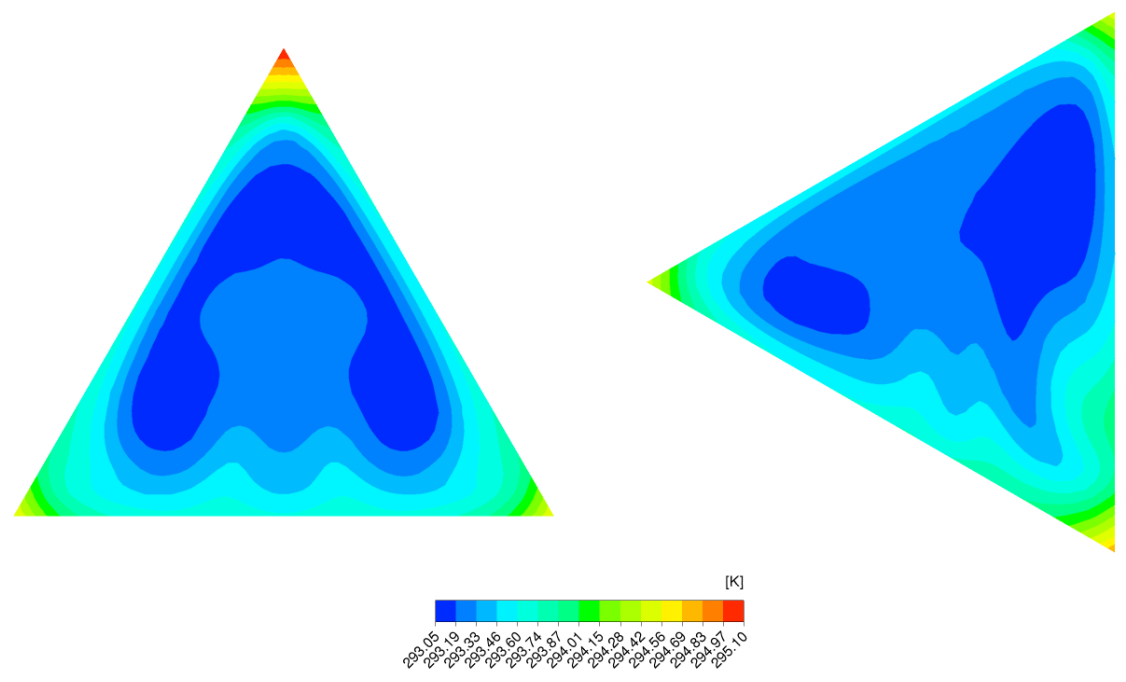

Figure 7: Temperature contours at the exit of Bend 2 of the SC-U (left) and SC-L (right) channels at Reynolds number of 150 .

Bend 1 of the SC-U geometry is observed to be slightly superior to that in the SC-L case. Additionally, the SC-U channel allows for lower heat transfer enhancement relaxation as the flow enters into Bend 4, and the effect becomes more pronounced with increment of the Reynolds number (see Figure 5).

To keep the cross-section at more favorable orientation for the heat transfer performance, it was varied along the SC-U channel by twisting, as shown in Figure 2. Figure 8 compares the variation of heat transfer enhancement across the SC-U and TSC-60 configurations for different Reynolds numbers. The changed orientation provides a remarkable heat transfer improvement already at $R e=50$; the heat transfer enhancement at the exit of Bend 3 is more than $35 \%$ greater compared to that of the SC-U, showing two-fold enhancement relative to the corresponding straight channel. The heat transfer enhancement is also larger in Bend 2 of the TSC-60 channel, and the performance difference becomes more pronounced at the higher Reynolds numbers. Thus, the heat transfer augmentation in Bend 2 and the following straight section of the TSC-60 configuration 


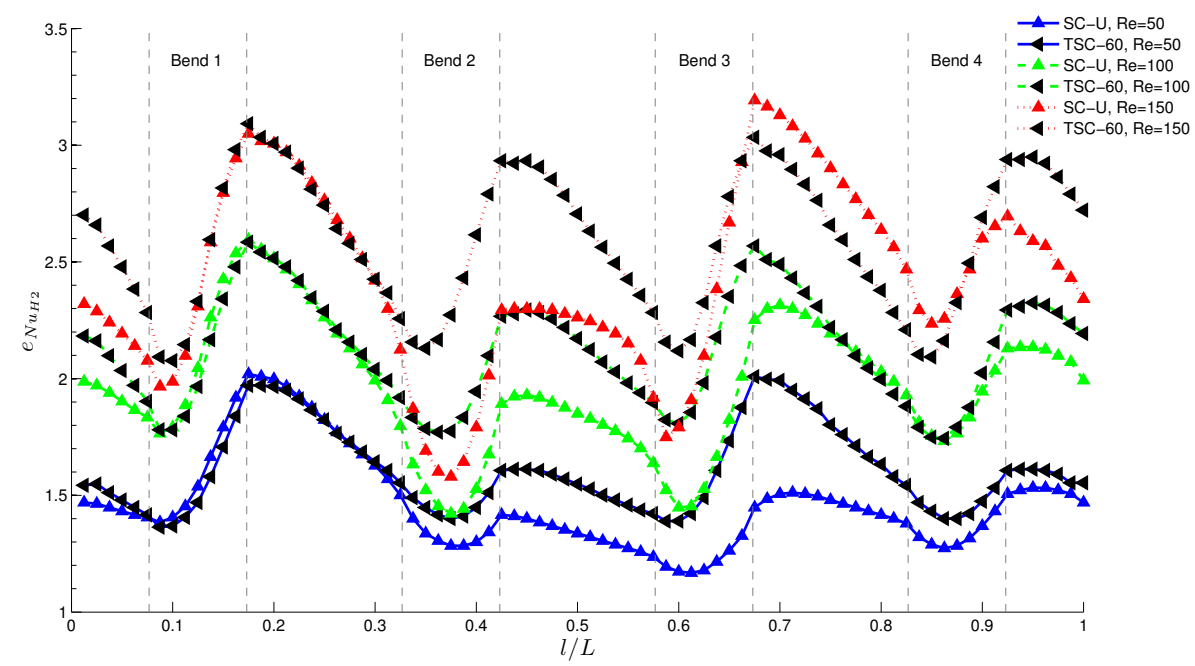

Figure 8: Heat transfer enhancement in the SC-U and TSC-60 configurations as a function of normalized axial distance at Reynolds numbers of 50,100 and 150. The intervals bounded by a pair of dashed vertical lines correspond to bend sections.

at $R e=100$ is comparable with that of the $\mathrm{SC}-\mathrm{U}$ at $R e=150$. Furthermore, at a Reynolds number of 150, the peak value of $e_{\mathrm{Nu}_{\mathrm{H} 2}}$ at the exit of Bend 2 of the TSC-60 channel is about 30\% higher than in the SC-U channel. However, at the same Reynolds number, the SC-U geometry still gives a slightly better heat transfer enhancement at the exit of Bend 3 compared to the twisted configuration. In Bend 4, the TSC-60 configuration outperforms the SC-U configuration in terms of heat transfer efficiency at all the $R e$ values considered, whereas the performance is nearly equal in Bend 1.

The change of the cross-section orientation thus gives rise to the heat transfer enhancement in Bends 2-4. Colder fluid is brought to the walls more effectively in the TSC-60 geometry, see Figure 9 and Figure 10. The intensity of the vortices in the SC-U channel becomes stronger only at the exit of Bend 3 at $R e=150$, providing a $5 \%$ higher peak heat transfer augmentation than with the TSC-60 configuration. Nevertheless, in the latter case, the overall heat transfer performance in Bend 3 is compensated by the lower relaxation of the heat transfer enhancement, as can be observed in Figure 8. The mean heat 

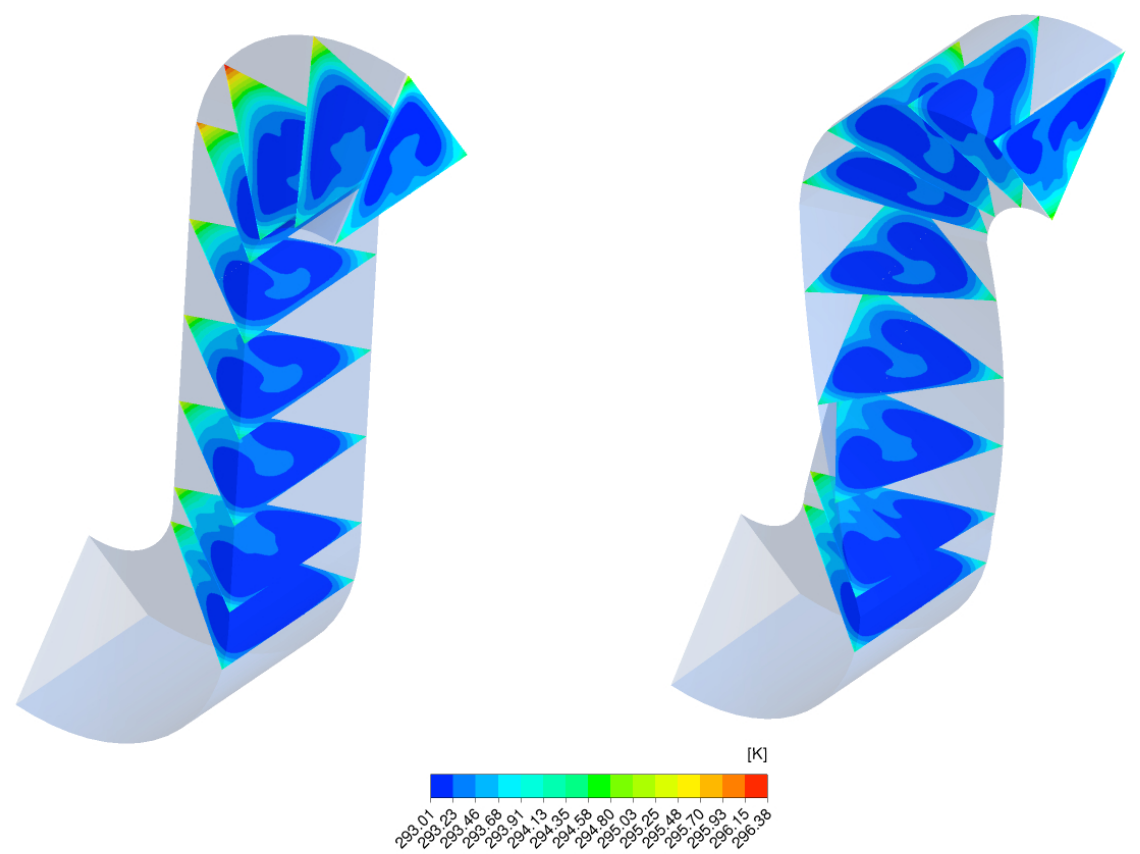

Figure 9: Temperature contours inside the SC-U (left) and TSC-60 (right) channels (Bend 2 and the upstream vertical section) at Reynolds number of 150 .

transfer enhancement for the TSC-60 channel is $9.5 \%, 8.2 \%$ and $7 \%$ greater than for the SC-U geometry at Reynolds numbers of 50, 100 and 150 respectively, and the twisted sections increase the pressure drop penalty only by $1.8 \%, 3.1 \%$ and $3.5 \%$.

The TSC-180 and the TSC-300 channels with a tighter twisting are next considered. The variation of heat transfer enhancement across the twisted configurations is shown in Figure 11. Heat transfer performance in the twisted sections reduces significantly with increasing degree of twisting. This occurs due to changes in the vortex structure. The Dean vortices formed in the bends usually advance into the following straight section and disappear after some distance further downstream. However, as can be seen in Figure 12, the twisted shape of the channel disrupts the vortex structure. The tighter twisted sections 


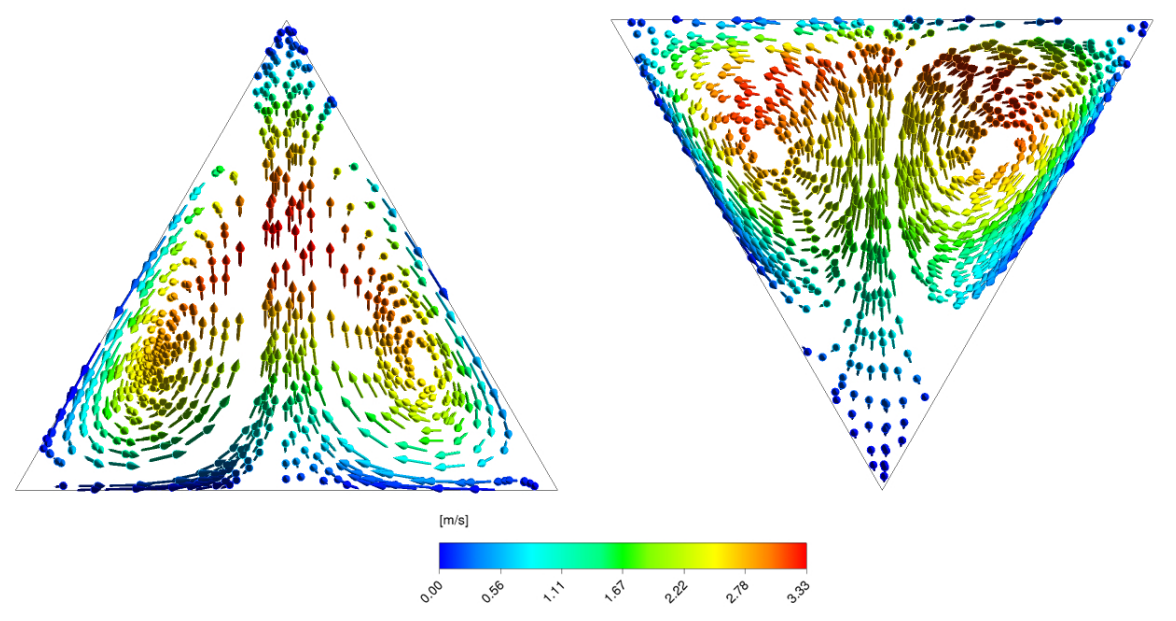

Figure 10: Velocity vectors colored by velocity magnitude at the exit of Bend 2 of the SC-U (left) and TSC-60 (right) channels at Reynolds number of 150.

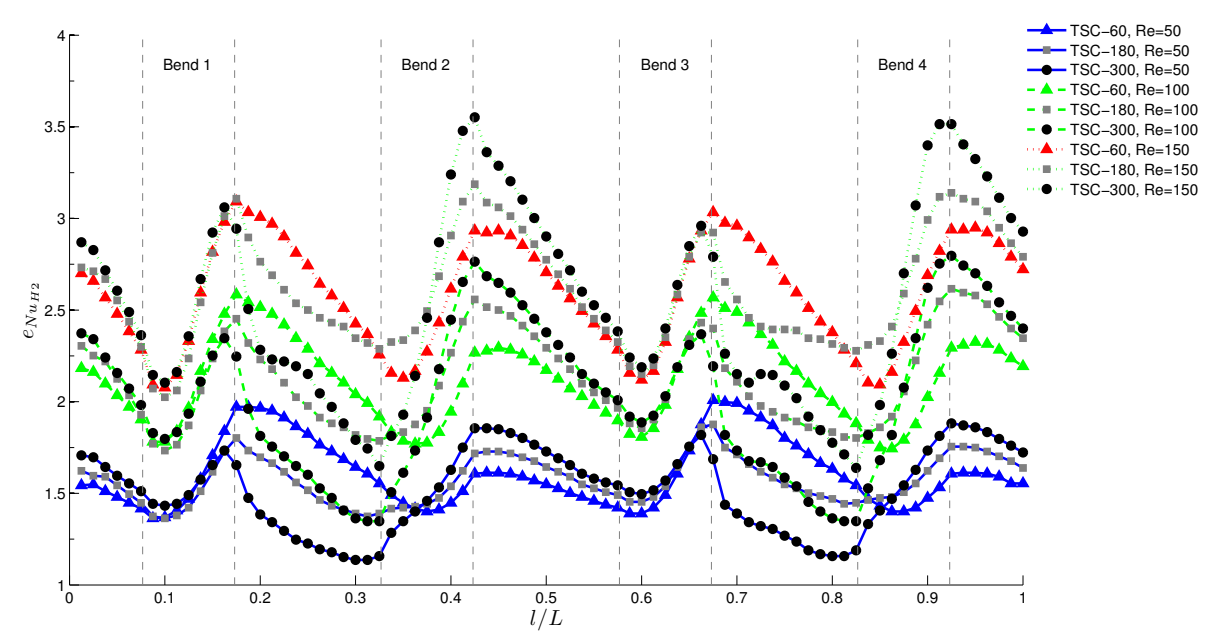

Figure 11: Heat transfer enhancement in the TSC-60, TSC-180 and TSC-300 configurations as a function of normalized axial distance at Reynolds numbers of 50, 100 and 150 . The intervals bounded by a pair of dashed vertical lines correspond to bend sections.

disrupt the Dean vortices earlier, see Figure 12a. Hence, the decline of $e_{N u_{H 2}}$ in the twisted sections increases with the degree of twisting and becomes more pronounced at higher Re values. In Figure 12b, a secondary flow can be detected 

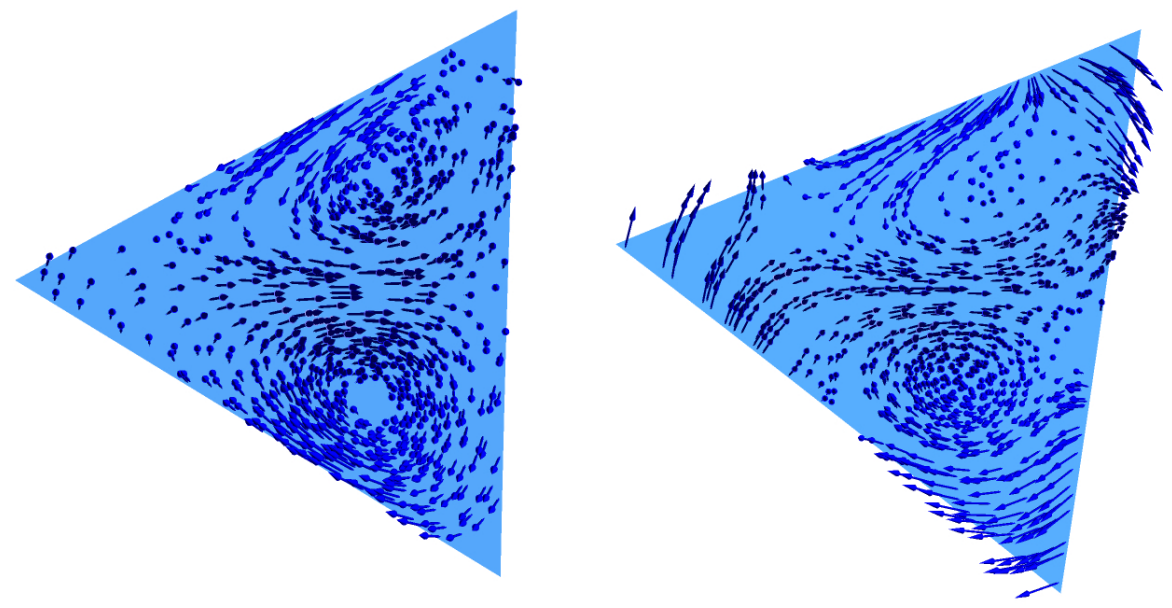

(a)
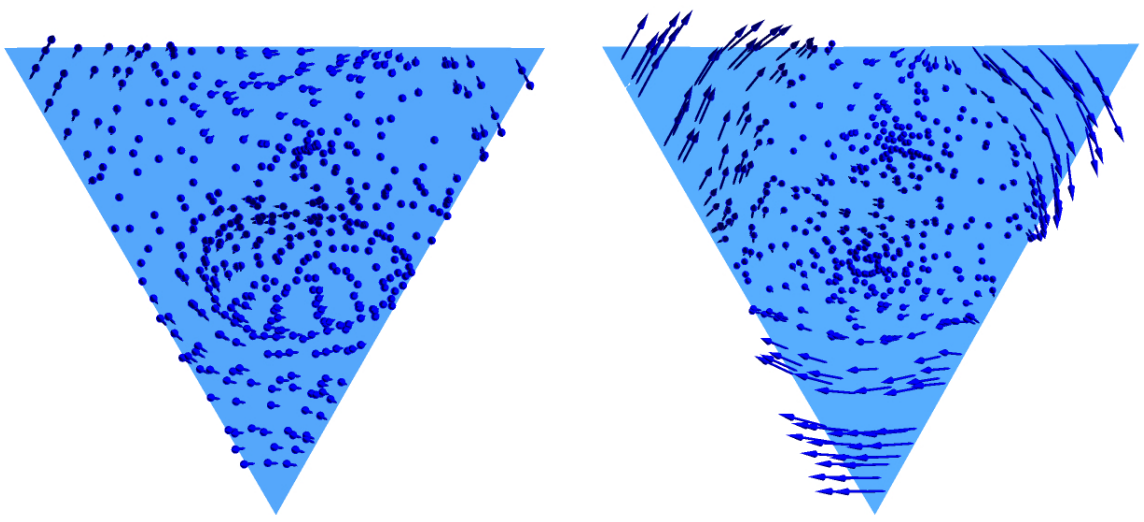

(b)

Figure 12: Secondary flows at the entrance (a) and the middle (b) of the twisted section (between Bend 1 and Bend 2) of the TSC-60 (left) and TSC-300 (right) channels at Reynolds number of 150

in the corners. This secondary flow is caused by the twisted shape, and has a swirling nature, as illustrated by the flow streamlines in Figure 13. Stronger twisting of the channel normally intensifies the secondary flow and thereby promotes the heat transfer $[14,17]$. With the configurations presented in this work, 


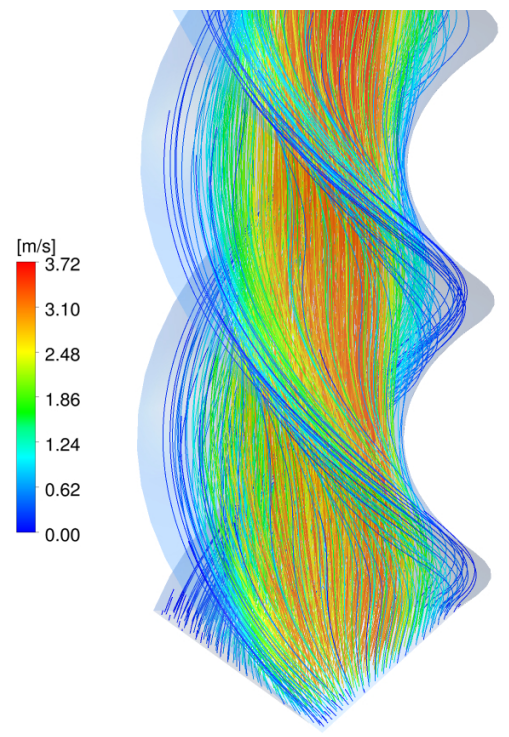

Figure 13: Streamlines colored by velocity magnitude inside the TSC-300 channel (twisted section between Bend 1 and Bend 2) at Reynolds number of 150 .

however, the opposite effect is observed.

According to [26], the heat transfer enhancement factor for a twisted channel with triangular cross-section decreases with the increment in Reynolds number. Therefore, a stronger attenuation of heat transfer enhancement in the twisted sections at the greater $R e$ values can be conditioned also by the cross-section shape. However, it is interesting to note that the results of Khoshvaght-Aliabadi and Arani-Lahtari [17] showed that Nusselt number for the same channel configuration increases with Reynolds number. The difference between the findings of [26] and [17] might be related to the channel geometry being twisted around different axes, that is, the center of rotation of the cross-section differs for the geometries studied. For example, in the case of a triangular cross-section, if the center of rotation does not coincide with the circumcenter, axial tortuosity of the channel appears, which promotes the heat transfer. Hence, when a twisted channel is designed, the center of rotation of the cross-section should be taken into account. 
In Figure 11, the peak heat transfer enhancement in the bends downstream of the twisted sections increases with the degree of twisting. In addition to the cross-section orientation, such augmentation can also be attributed to the swirling flow caused by the preceding twisted sections. Moreover, the flow enters these bends with a slightly higher peak axial velocity as the degree of twisting increases, see Figure 14. The difference between the heat transfer enhancement
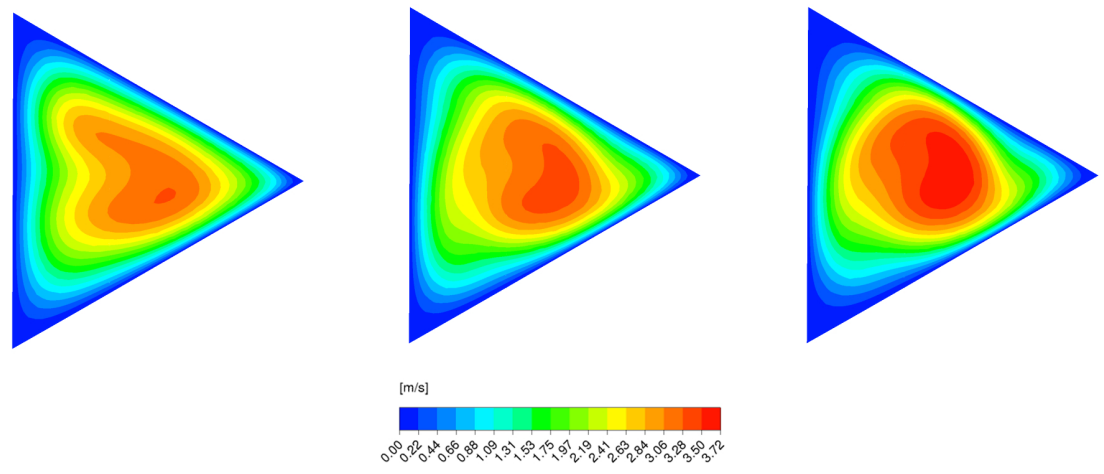

Figure 14: Velocity distribution at the entrance of Bend 2 (similarly for Bend 4) of the TSC-60 (left), TSC-180 (center) and TSC-300 (right) channels at Reynolds numbers of 150.

at the entrance and the exit of the bend just after the twisted section can thus be considerable. For instance, for the TSC-300 channel at $R e=150$, the heat transfer augmentation at the exits of Bend 2 and Bend 4 is more than 3.5 times higher than in the corresponding straight channel, whereas the enhancement at the entrances barely reaches the value of 1.6.

The estimated mean heat transfer enhancements and pressure drop penalties for the studied channels at different $R e$ values are summarized in Figure 15. It can be seen that the TSC-180 and TSC-300 configurations do not provide a superior mean heat transfer enhancement than the TSC-60 configuration. With the TSC-180 geometry, $\bar{e}_{N u_{H 2}}$ decreases by $2.5 \%$ at $R e=50$, and stays at the same level as the TSC-60 configuration at Reynolds numbers of 100 and 150 . The mean heat transfer enhancement factor for the TSC-300 configuration shows $3.2 \%, 4.7 \%$ and $2.7 \%$ lower values than for the TSC-180 channel at Reynolds 


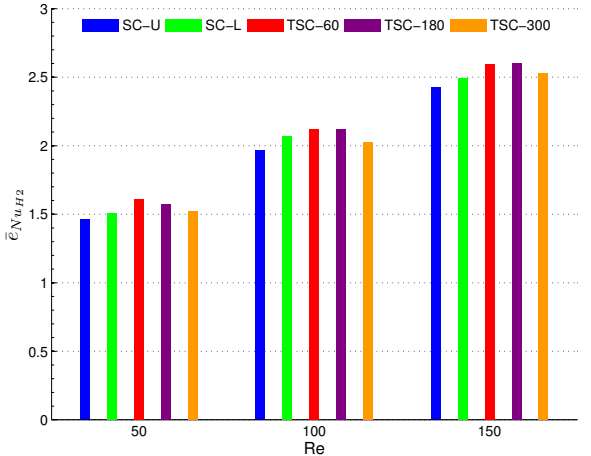

(a)

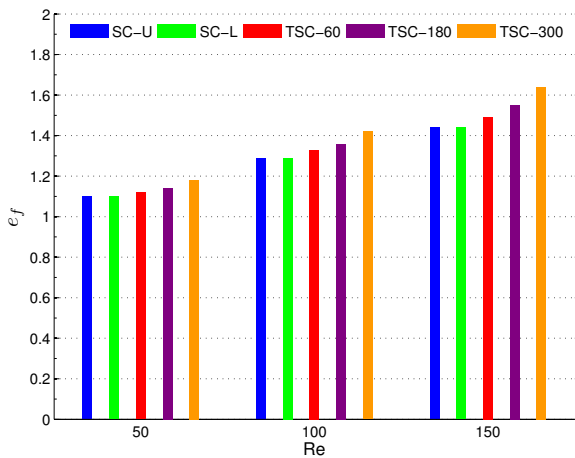

(b)

Figure 15: Mean heat transfer enhancement (a) and pressure drop penalty (b) for different channel configurations at Reynolds numbers of 50,100 and 150.

numbers of 50, 100 and 150, respectively. Moreover, at $R e=100$, the mean heat transfer enhancement for the TSC-300 geometry is $2.4 \%$ lower relative to that for the SC-L.

The pressure drop increases with the Reynolds number for all the configurations. Among the considered cases, the SC-U and SC-L geometries have the smallest pressure drop penalty. At $R e=50$, the pressure drop in the these configurations is only $10 \%$ higher than in the corresponding straight channel. However, with increase of the Reynolds number to 150, the pressure drop increases further by more than $30 \%$ to 1.44 . For the channels with twisted sections, the pressure drop rises also with degree of twisting due to increasing intensity of the swirling flow. Compared to the SC-U geometry, the pressure loss exhibited by the TSC-300 configuration is $7.3 \%, 10.1 \%$ and $13.9 \%$ greater at $R e=50,100$ and 150 respectively.

The overall performance of each channel based on the PEC is presented in Figure 16. For all the channels, the PEC increases with the Reynolds number. At Reynolds number of 50, the TSC-60 channel provides a noticeably higher value for $E$ than the other ones, followed by the TSC-180 channel with $E$ of 1.51. At $R e=100$, having almost equal overall performances, the SC-L, TSC-60 


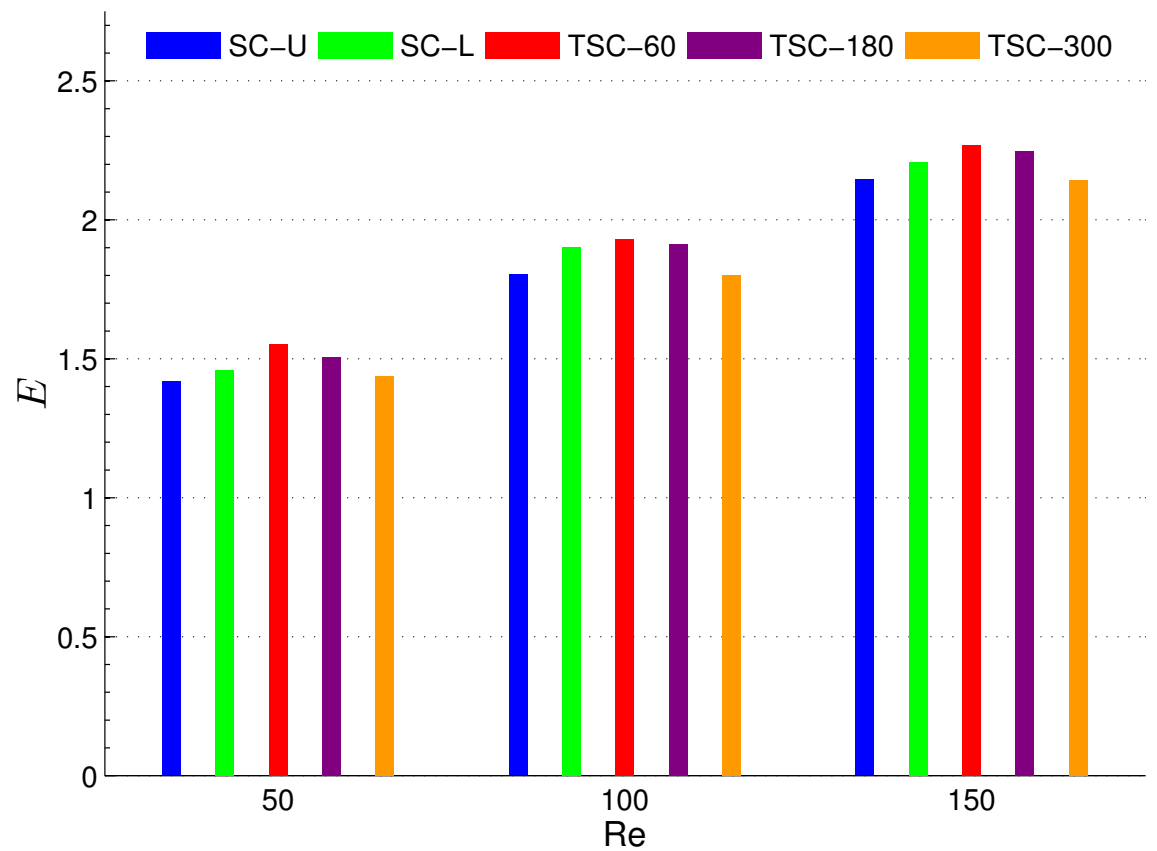

Figure 16: PEC for different channel configurations at Reynolds numbers of 50, 100 and 150.

and TSC-180 geometries outperforms the other configurations in terms of the PEC. The maximum $E$ of about 2.3 is achieved by the TSC-60 configuration at $R e=150$. The SC-U and TSC-300 channels give the least PEC at all the Reynolds numbers studied.

\section{Conclusions}

The effect of cross-section orientation on the thermal performance of a serpentine microchannel with an equilateral triangular cross-section was studied numerically. The fluid flow and heat transfer were simulated in Fluent 15.0 under the constant wall heat flux (H2) thermal boundary conditions at Reynolds numbers of 50, 100 and 150 . The local heat transfer was shown to be sensitive to the cross-section orientation and heat transfer can be improved by changing the orientation. With the same pressure drop, the SC-L configuration demonstrated a higher mean heat transfer enhancement than the SC-U geometry at 
all the Reynolds numbers considered. Serpentine channel configurations with twisted sections were also considered and evaluated. Variation of the crosssection orientation by twisting had a significant impact on heat transfer and fluid flow. The TSC-60 channel showed a noticeably better mean heat transfer enhancement than the non-twisted configurations and the pressure loss increase was insignificant.

Tighter twisting of the sections did not provide a higher mean heat transfer enhancement factor and resulted in greater pressure drop. At $R e=50$, the mean heat transfer enhancement for the TSC-180 channel decreased slightly but remained equal at the higher $R e$ values. The mean heat transfer enhancement for the TSC-300 also reduced slightly further at all the Reynolds numbers studied, compared to the TSC-180. The heat transfer enhancement variation along the 340 TSC-180 and TSC-300 channels was considerable. The twisted sections tended to disrupt the Dean vortices formed in the upstream bends, substantially deteriorating the heat transfer performance, whereas the effect was negligible in the TSC-60 configuration due to weaker twisting. At the same time, stronger twisting gave a remarkable increase in the heat transfer enhancement in the downstream bends due to the the swirling flow generated by the twisted geometry. It is concluded that utilization of twisted shapes is capable of improving heat transfer performance of serpentine channels.

\section{Acknowledgments}

The authors wish to acknowledge CSC-IT Center for Science, Finland, for 350 providing computational resources.

\section{References}

[1] S. G. Kandlikar, Heat Transfer and Fluid Flow in Minichannels and Microchannels, Elsevier, Amsterdam, Netherlands San Diego, CA Oxford, UK, 2006. 
[2] M. E. Steinke, S. G. Kandlikar, Review of single-phase heat transfer enhancement techniques for application in microchannels, minichannels and microdevices, International Journal of Heat and Technology 22 (2) (2004) $3-11$.

[3] L. Léal, M. Miscevic, P. Lavieille, M. Amokrane, F. Pigache, F. Topin, B. Nogarède, L. Tadrist, An overview of heat transfer enhancement methods and new perspectives: Focus on active methods using electroactive materials, International Journal of Heat and Mass Transfer 61 (2013) 505524.

[4] A. M. Adham, N. Mohd-Ghazali, R. Ahmad, Thermal and hydrodynamic analysis of microchannel heat sinks: A review, Renewable and Sustainable Energy Reviews 21 (2013) 614-622.

[5] I. A. Ghani, N. A. C. Sidik, N. Kamaruzaman, Hydrothermal performance of microchannel heat sink: The effect of channel design, International Journal of Heat and Mass Transfer 107 (2017) 21-44.

[6] P. E. Geyer, D. F. Fletcher, B. S. Haynes, Laminar flow and heat transfer in a periodic trapezoidal channel with semi-circular cross-section, International Journal of Heat and Mass Transfer 50 (17) (2007) 3471-3480.

[7] C. M. Karale, S. S. Bhagwat, V. V. Ranade, Flow and heat transfer in serpentine channels, AIChE Journal 59 (5) (2013) 1814-1827.

[8] Y. Sui, C. Teo, P. Lee, Y. Chew, C. Shu, Fluid flow and heat transfer in wavy microchannels, International Journal of Heat and Mass Transfer 53 (13) (2010) 2760-2772.

[9] H. Mohammed, P. Gunnasegaran, N. Shuaib, Numerical simulation of heat transfer enhancement in wavy microchannel heat sink, International Communications in Heat and Mass Transfer 38 (1) (2011) 63-68. 
[10] N. R. Rosaguti, D. F. Fletcher, B. S. Haynes, Laminar flow and heat transfer in a periodic serpentine channel, Chemical Engineering \& Technology 28 (3) (2005) 353-361.

[11] P. E. Geyer, N. R. Rosaguti, D. F. Fletcher, B. S. Haynes, Thermohydraulics of square-section microchannels following a serpentine path, Microfluidics and Nanofluidics 2 (3) (2006) 195-204.

[12] R. Gupta, P. E. Geyer, D. F. Fletcher, B. S. Haynes, Thermohydraulic performance of a periodic trapezoidal channel with a triangular cross-section, International Journal of Heat and Mass Transfer 51 (11) (2008) 2925-2929.

[13] R. J. Silva, R. M. Valle, M. Ziviani, Numerical hydrodynamic and thermal analysis of laminar flow in curved elliptic and rectangular ducts, International Journal of Thermal Sciences 38 (7) (1999) 585-594.

[14] X.-H. Tan, D.-S. Zhu, G.-Y. Zhou, L.-D. Zeng, Experimental and numerical study of convective heat transfer and fluid flow in twisted oval tubes, International Journal of Heat and Mass Transfer 55 (17) (2012) 4701-4710.

[15] R. Bhadouriya, A. Agrawal, S. Prabhu, Experimental and numerical study of fluid flow and heat transfer in a twisted square duct, International Journal of Heat and Mass Transfer 82 (2015) 143-158.

[16] S. Yang, L. Zhang, H. Xu, Experimental study on convective heat transfer and flow resistance characteristics of water flow in twisted elliptical tubes, Applied Thermal Engineering 31 (14) (2011) 2981-2991.

[17] M. Khoshvaght-Aliabadi, Z. Arani-Lahtari, Forced convection in twisted minichannel (TMC) with different cross section shapes: A numerical study, Applied Thermal Engineering 93 (2016) 101-112.

${ }_{405}$ [18] M. Khoshvaght-Aliabadi, Z. Arani-Lahtari, Proposing new configurations for twisted square channel (TSC): Nanofluid as working fluid, Applied Thermal Engineering 108 (2016) 709-719. 
[19] Z. Dai, D. F. Fletcher, B. S. Haynes, Impact of tortuous geometry on laminar flow heat transfer in microchannels, International Journal of Heat and Mass Transfer 83 (2015) 382-398.

[20] S. Patankar, C. Liu, E. Sparrow, Fully developed flow and heat transfer in ducts having streamwise-periodic variations of cross-sectional area, Journal of Heat Transfer 99 (2) (1977) 180-186.

[21] R. K. Shah, Laminar Flow Forced Convection in Ducts : A Source Book for Compact Heat Exchanger Analytical Data, Academic Press, New York, 1978.

[22] Ansys, Inc, Canonsburg, PA, ANSYS FLUENT Theory Guide (2011).

[23] Ansys, Inc, Canonsburg, PA, ANSYS FLUENT User's Guide (2011).

[24] R. Webb, Performance evaluation criteria for use of enhanced heat transfer surfaces in heat exchanger design, International Journal of Heat and Mass Transfer 24 (4) (1981) 715-726.

[25] W. Dean, XVI. Note on the motion of fluid in a curved pipe, The London, Edinburgh, and Dublin Philosophical Magazine and Journal of Science 4 (20) (1927) 208-223.

[26] M. Khoshvaght-Aliabadi, Z. Arani, F. Rahimpour, Influence of Al2O3$\mathrm{H} 2 \mathrm{O}$ nanofluid on performance of twisted minichannels, Advanced Powder Technology 27 (4) (2016) 1514-1525. 